\title{
Specific activation of embryonic IFNAR1 and endometrial IFNAR2 induced by embryonic IFNT directs normal uterine fate for bovine early implantation
}

\section{Yong Guo ( $\nabla$ y63guo@126.com )}

Beijing University of Agriculture

\section{Xiangguo Wang}

Beijing University of Agriculture

\section{Chaolei Chen}

Beijing University of Agriculture

\section{Lijuan Wang}

Beijing University of Agriculture

\section{Yunze Su}

Beijing University of Agriculture

Boyu Li

Beijing University of Agriculture

\section{Longfei Xiao}

Beijing University of Agriculture

\section{Zili Lin}

Beijing University of Agriculture

Xihui Sheng

Beijing University of Agriculture

Xiaolong Qi

Beijing University of Agriculture

Hemin Ni

Beijing University of Agriculture

\section{Research Article}

Keywords: Bovine, blastocyst, IFNt, endometrial cell, IFNAR

Posted Date: February 1st, 2022

DOI: https://doi.org/10.21203/rs.3.rs-1297654/v1 
License: (c) (i) This work is licensed under a Creative Commons Attribution 4.0 International License. Read Full License 


\section{Abstract}

Background: Interferon-tau (IFNT), as an antiluteolytic factor secreted by trophoderm during the

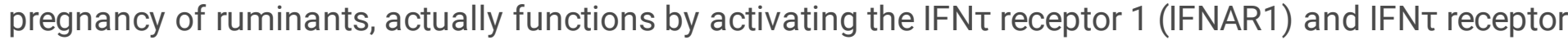
2 (IFNAR2). However, it has not been clearly understood how IFNT-IFNAR cascade regulation processes between the embryo and uterine epithelial cells in ruminants.

Methods: CRISPR/Cas9 technology was used to compare the differences of IFNt secretion in In vitro fertilization(IVF), parthenogenetic activation(PA) and somatic cell nuclear transfer (SCNT) and their in vitro co-culture effect with uterine endometrial epithelial cell with those in vivo ones in this study.

Results: In this study, we found the expression and location of IFN $\tau$ in the blastocysts from different sources. IFNT, IFNAR1 and IFNAR2 were all located in the trophoblast cells of the blastocyst. However, the fluorescence intensity of IFNAR1 was consistent with that of IFNT. Antagonizing the expressions of IFNAR1 and IFNAR2 in embryos and co-culture with EEC reduced the expressions of Integrin av $\beta 3$, WNT7A, and ISG15 in EECs. Knocking out IFNAR1 and IFNAR2 reduce the expressions of Integrin av $\beta 3$ and WNT7A in EECs, the deletion of IFNAR2 gene has a greater impact than that of IFNAR1 gene. IFNAR $1^{-} /$IFNAR2 ${ }^{+}$and IFNAR $1^{+} /$IFNAR2 ${ }^{-}$EECs were co-cultured with IVF embryos, the expression of Integrin av $\beta 3$ was inhibited, and the inhibition of IFNAR1 ${ }^{+} /$IFNAR2 $2^{-}$was much stronger, and the expression of WNT7A was not inhibited. The expressions of Integrin av $\beta 3$ and WNT7A did not change significantly after IFNAR1/IFNAR2 ${ }^{+}$and IFNAR1 ${ }^{+} /$IFNAR2 ${ }^{-}$co-culture with PA embryos.

Conclusions: All of these results strongly suggest that specific activation of embryonic IFNAR1 and endometrial IFNAR2 induced by embryonic IFNT directs normal uterine preparation for bovine early implantation.

\section{Background}

For the purpose of the precise recognition dialogue between the maternal uterus and the early embryo, the normal mammalian embryo in the active state must be synchronized with the uterus in the tolerant state in time and space [1-5]. Up to now, there have been many reports on the reproductive mechanism of early pregnancy of mammals, and it was found that the factors involved in the precise regulation of early pregnancy are not exactly the same in different animals [1-4, 6]. Estrogen (E2), progesterone (P4), various factors and enzymes are involved in the precise regulation of the pregnancy of their early embryos in vivo. However, these various factors are involved in the precise regulation of early pregnancy in ruminants, such as cattle and sheep, and there are also obvious differences in many other key regulatory factors between the large ruminants represented by cattle and these classic experimental animals, such

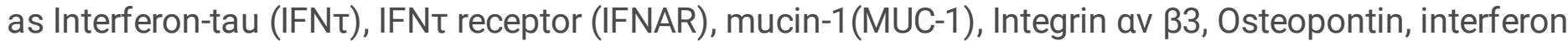
stimulated gene 15 (ISG15), and highly conserved secreted WNT family subtype glycoprotein (WNT). Among them, IFNt is currently the only known signaling protein produced by the embryo, which induces the production of WNT7A and Integrin av $\beta 3$ protein in the maternal uterus whose synergistic effect is the 
normal initiation of bovine embryos in combination with its specific receptor IFNAR, and plays a role of the most critical regulator in maintaining their normal pregnancy $[4,5,7-10]$.

As a specific receptor for IFNT and special receptor protein shared by interferon family proteins, IFNAR is usually composed of two subunits, i.e., IFNAR1 and IFNAR2, which regulate downstream WNT7A, Integrin av $\beta 3$ and other specific binding reactions by means of anchor binding to certain special proteins in cell membranes, and takes part in the regulation of early pregnant initiation in ruminant livestock $[5,8,9,11$, 12]. Studies have shown that the recombinant IFNT is used to perfuse the ovine uterus during the natural estrus or on the 14th and 15th day after intercourse. Both IFNAR1 and IFNAR2 were expressed in luminal epithelial cells and glandular epithelial cells [11]. Another study found that the expressions of IFNAR1 and IFNAR2 were not consistent in bovine placentas during early pregnancy. IFNAR1 mainly distributed in chorioallantoic membrane, endometrial epithelium, cotyledon and caruncle and differed from time and position during the early pregnancy of cattle. The expressions of IFNAR1 and IFNAR2 were mainly detected in chorioallantoic membrane and cotyledon, and markedly increased along with the pregnant process. Moreover, the expression level of IFNAR1 was higher than that of IFNAR2 in chorioallantoic membrane and cotyledon [13]. In addition, the research confirmed that IFNAR2 plays an important role in regulating immune function and HIV invasion $[14,15]$ and IFNAR1 does in the immune protection of various vaccines in vivo [16]. Therefore, it is crucial for understanding the role of IFN $\tau$ and its receptors in keeping normal early implantation of ruminants to ascertain the different expression patterns of IFNAR1 and IFNAR2.

At present, the research on the reproduction mechanism of large livestock focuses on the implantation process of early animal embryos obtained with the in vitro manipulation methods such as superovulation, in vitro production, and somatic cell cloning $[2,3,6,12]$. However, embryos prepared in vitro differ in quality from those produced by the natural mating of the mother under normal circumstances. Existing studies have found that the moderate addition of recombinant IFN $\tau$ in culture fluid in vitro can increase the blastocyst's development rate in bovine IVF embryos [17]. Therefore, it will fundamentally provide a new reference for further improving their pregnant rate to make in-depth discussion about IFNT secreted by ruminant livestock's embryos through its specific receptor IFNAR to regulate the precise embryo-endometrium dialogue. However, it has not been known clearly how the IFNTIFNAR cascade regulation processes between the embryo and uterine epithelial cells $[4,5,9,17]$.

So far, the technologies of in vitro fertilization (IVF), parthenogenetic activation (PA) and somatic cell nuclear transfer (SCNT) have been applied to obtaining in vitro blastocysts, and those in vivo blastocysts produced by superovulation and natural mating were used to compare the differences in their own IFN secretion and their co-culture with EECs in this study, respectively. The differences between the regulation mechanism of the IFNAR cascade of embryos and that of the EECs of uterine receptive proteins were researched to further dig out the process of the early implantation in vitro of bovine embryos. The autocrine method binds to and functions with IFNAR to regulate the molecular mechanism of precise embryo-uterine dialogue, for which we analyzed its relationship with implantation failure, early abortion 
and other reproductive diseases so as to ultimately further improve the embryo-transfer pregnancy rate of the embryos in vitro produced in ruminants.

\section{Methods}

\section{Isolation, culture and identification of primary endometrial cells of dairy cows}

This study was performed in accordance with the Guidelines of the Animal Ethic Committee of Beijing University of Agriculture under the permit No. SYXK (JING) 2015-0004. The uterus of healthy cows was taken from the slaughterhouse, stored in DPBS containing $4 \%$ penicillin and transported to the laboratory at $37^{\circ} \mathrm{C}$. Then the tissue was cut to select the uterus-free carcass tissue, which was then washed with DPBS for 2-3 times and cut into small pieces. The endometrial epithelial cells (EECs) were isolated with the tissue migration method, and passed through 3-4 passages successively. Fibroblasts were removed with $0.25 \%$ pancreatin and differentially digested at $37^{\circ} \mathrm{C}$. The purity of EECs was identified by detecting the expression of keratin 7 protein with the immunofluorescence method.

\section{In vitro maturation of oocytes}

The bovine ovaries were taken from the slaughterhouse, placed in the cleaning solution of penicillin and streptomycin, transported back to the laboratory within $2 \sim 4 \mathrm{~h}$, and washed repeatedly in the laboratory. Then a $20 \mathrm{~mL}$ syringe was used to aspirate the follicular fluid from a follicle with a diameter of 3 to $8 \mathrm{~mm}$ and pour it into a $60 \mathrm{~mm}$ Petri dish. A high-quality cumulus-oocyte complex (The cumulus has three layers or more and is densely wrapped without diffusion) was selected under the microscope and washed for three times in the HEPES droplets, before it was put in the pre-balanced IVM mature droplets, washed for three times, and transferred to and incubated in an incubator with $5 \% \mathrm{CO}_{2}$ at $38.5^{\circ} \mathrm{C}$ for $22 \mathrm{~h}$.

\section{Parthenogenetic activation}

The in vitro matured oocytes, $1 \mathrm{~mL}$ of HEPES egg wash, and $200 \mu \mathrm{L}$ of $0.1 \%$ hyaluronidase were all added to a $10 \mathrm{~mL}$ centrifuge tube which then was shaken with a shaker for $4 \mathrm{~min}$. The granulosa cells around the mature oocytes were removed. Next, the oocytes that have been expelled from the first polar body were picked out and moved into the ION droplets to protect them from light for $5 \mathrm{~min}$. After activation, it was transferred into the 6-DMAP droplets, washed twice, then cultured in the 6-DMAP droplets for $4 \mathrm{~h}$, and finally transferred into the embryo culture medium which was cultured in a saturated humidity environment with $5 \% \mathrm{CO}_{2}$ at $38.5^{\circ} \mathrm{C}$. The embryo culture medium ( $\mathrm{mCR}$ ) containing $10 \% \mathrm{FBS}$ was changed in half every $48 \mathrm{~h}$, and the cleavage rate was counted after $48 \mathrm{~h}$ after the parthenogenetic activation operation. The day when the parthenogenetic activation started was set to the zeroth day, and the blastocyst rate was counted till the 7th day. 


\section{In vitro fertilization}

After in vitro maturation, the oocytes were washed twice with the HEPES egg wash, and then moved into the pre-balanced fertilization droplets. Each drop of 10 15 oocytes was combined with 1 2 pieces of frozen semen (Beijing Dairy Cow Center: Limousin (No. 11108016)), shaken in the air for $10 \mathrm{~s}$, and then thawed in a $37^{\circ} \mathrm{C}$ water bath. The motility and density of sperms were checked. The thawed semen was slowly added to the sperm wash, and centrifuged at 1,800 rpm for 8 min twice. After the supernatant was discarded, the sperm concentration was adjusted to $2 \sim 4 \times 10^{6} / \mathrm{mL}$ for in vitro fertilization. The treated frozen semen was injected into the fertilized droplets containing $15 \mu \mathrm{L}$ mature oocytes per drop, which was placed in a saturated humidity incubator with $5 \% \mathrm{CO}_{2}$ at $38.5^{\circ} \mathrm{C}$. After the incubation of a total of 18 $h$, the early embryos after fertilization were separated from the fertilization fluid, and placed and shaken on a shaker. Next, the HEPES egg washing solution was added to the highest level of the shaker, and centrifuged at 1,000 rpm for $1 \mathrm{~min}$. The embryos sucked out of the tube were washed twice with HEPES and cultured in a saturated humidity environment with $5 \% \mathrm{CO}_{2}$ at $38.5^{\circ} \mathrm{C}$. The late embryo culture medium containing FBS was changed once every $48 \mathrm{~h}$. The cleavage rates of in vitro fertilized embryos were counted after $48 \mathrm{~h}$ after fertilization. The day when fertilization started was set to the zeroth day, and the blastocyst rate was counted up to the 8th day.

\section{Somatic cell nuclear transfer}

The fresh bovine ears were collected from the slaughterhouse, and the tissue block migration method was used to obtain the bovine ear fibroblasts, and the microinjection method to drive the donor bovine ear fibroblasts into the enucleated oocytes to prepare the reconstructed embryos, which were then placed in the electrofusion tank, and activated with $250 \mathrm{~V} / \mathrm{cm}$ and 2DC fusion parameter alternating current for 10 $\mu s$. After the electrofusion, the embryos were put into the incubator with ION (ionomycin) in the dark for $5 \mathrm{~min}$, and finally transferred to the $100 \mu \mathrm{L} 6$-DMAP droplets which then were chemically activated in the incubator for $4 \mathrm{~h}$, transferred to M1 for cultivation for $48 \mathrm{~h}$, and finally replaced in half with M2 late culture.

\section{IFNAR-IN-1 hydrochloride (IN-1) concentration screening}

Eight-cell IVF embryos were incubated with $10 \mu \mathrm{M} I \mathrm{~N}-1$ to the blastocyst stage, and then with EECs for 48 h. Co-cultured EECs were collected, and total protein from the EECs was subjected to the detection of the expressions of IFNAR1, IFNAR2, Integrin av, Integrin $\beta 3$ and WNT7A with the western blot method, respectively. $\beta$-actin served as the control gene in the all samples. In addition, the EECs were cultured with $5 \mu \mathrm{M} \mathrm{IN}-1$ antagonist and $0.01 \mathrm{ng} / \mathrm{mL}$ IFNT for $24 \mathrm{~h}$. Then total mRNA from EECs was subjected to the detection of the expressions of ISG15 and IRF9 with the qRT-PCR method. 


\section{IVF embryos with INFAR1 and IFNAR2 knockout embryos obtained}

The designed sgRNA1-F, sgRNA2-F, sgRNA3-F, sgRNA4-F, and sgRNA-R (Table S1) were biosynthesized, amplified in vitro, purified by phenol/chloroform, and then combined with the whole genome of EECs after the amplification of sgRNA1, sgRNA2, sgRNA3, and sgRNA4 targets for spCas9 activity in vitro biopsy. RTPCR was used to detect any target cleavage activity. The effective sgRNA1 and sgRNA4 were microinjected into the embryo after $10 \mathrm{~h}$ of sperm-egg combination, and continued to develop to the blastocyst stage. After that, the blastocysts were incubated with EECs for $48 \mathrm{~h}$ and collected for T7E1 digestion reaction. RT-PCR was used to detect any genomic target cleavage activity.

\section{Screening of INFAR1 and IFNAR2 gene CRISPR/Cas9 knockout cell lines (IFNAR1-/IFNAR2-), (IFNAR1-/IFNAR2+) and (IFNAR1+/IFNAR2-)}

Three pairs of INFAR1 and IFNAR2 gRNA sequences (Table S2) were designed, and then ligated into the PX459 plasmid. Three dual-target plasmids were constructed as 11: sgRNA1-1 + sgRNA2-1; I2: sgRNA1-2 + sgRNA2-2; 13: sgRNA1-3 + sgRNA2-3. Sanger sequencing was performed with U6 primers. The sequencing results confirmed that the two sgRNA fragments in the three plasmids had been ligated to the PX459 plasmid. The INFAR1 and IFNAR2 dual-target recombinant vector was transfected into the EECs with Lipofectamine 3000. After puromycin selection, the picked monoclonal cells were cultured by adding MEM to maintain the culture medium. When the confluence reached $80 \%-90 \%$, the EECs were collected to verify the expression of INFAR1 and IFNAR2 proteins with Western blot.

INFAR1 and INFAR2 gRNA sequences (Table S3) were designed, and then ligated them into the PX459 plasmid. To construct INFAR1 and INFAR2 target plasmids, we used U6 primers for Sanger sequencing the correct sequencing results of PX459-sgRNA3 (IFNAR1/IFNAR2 ${ }^{+}$) and PX459-sgRNA4 (IFNAR1 ${ }^{+} /$IFNAR2 ${ }^{-}$) which were then transfected into EEC with Lipofectamine 3000 and into EEC with puromycetes in turn. After the element screening, the picked monoclonal cells were cultured by adding MEM to maintain the culture medium. When the confluence reached $80 \%-90 \%$, the EECs were collected to verify the expression of INFAR1 and INFAR2 proteins with Western blot.

\section{Co-culture of blastocysts with EECs}

The purified and identified primary EECs were sub-cultured, and the bottom of the culture dish was fused into a single layer of cells. After the embryos were parthenogenetically activated, the in vitro fertilized embryos were harvested, the blastocysts (five blastocysts in each group) obtained in vivo and somatic nuclear transfer blastocysts were placed in a single layer of EECs and co-cultured for $24 \mathrm{~h}$. Finally, the embryos and EECs were collected for later use. 


\section{RNA extraction and RT-PCR}

The samples of blastocyst and EECs were collected separately. Total RNA was extracted with Trizol reagent (Invitrogen) from the fresh tissues according to the manufacturer's protocol, followed by the removal of genomic DNA with RNase-free DNase (Promega, Madison, WI). After 15 reverse transcriptions, IFNT, ISG15, WNT7A, IFNAR1, IFNAR2 and $\beta$-actin were amplified with PCR. The PCR reactions were conducted in a total volume of $10 \mu \mathrm{L}$. The target gene was amplified for 30 cycles of denaturation at $94^{\circ} \mathrm{C}$ for $30 \mathrm{~s}$, annealed at $57^{\circ} \mathrm{C}$ for $30 \mathrm{~s}$, and extended at $72^{\circ} \mathrm{C}$ for $30 \mathrm{~s}$. This was followed by a final $5 \mathrm{~min}$ extension step at $72^{\circ} \mathrm{C}$. The amplified products were analyzed with electrophoresis on $1.5 \%$ agarose gels stained with ethidium bromide. The primers used for the analysis were shown in Table S4.

\section{Real-time detection of RT-PCR (qRT-PCR)}

Total RNA of each treated cell was extracted with the Trizol (Invitrogen, Inc., Carlsbad, CA, USA) and cDNA was synthesized with the PrimeScript ${ }^{\mathrm{TM}} \mathrm{RT}$ Reagent Kit (TaKaRa Bio, Inc., Dalian, China), according to the manufacturers' protocols, respectively. Real-time PCR was subsequently performed with an ABI 7500 Sequencing Detection System and SYBR Premix Ex Taq ${ }^{\text {TM }}$. The GenBank accession numbers and primer sequences of ISG15, IRF9, Integrin av, Integrin $\beta 3$, WNT7A and GAPDH are summarized in Table S5. All reactions were performed in at least three independent experiments and the calculated number of copies of target genes was normalized to the number of GAPDH mRNA copies in the same sample.

\section{Immunofluorescence}

The blastocysts or EECs were fixed in $4 \%$ PFA at $4^{\circ} \mathrm{C}$ for $24 \mathrm{~h}$. Subsequently, the cells were permeabilized with $0.1 \%$ TritonX-100 for $10 \mathrm{~min}$, blocked with $5 \%$ BSA for $30 \mathrm{~min}$, and exposed to anti-IFNt, IFNAR1, IFNAR2 and Keratin primary antibody (1:1000 diluted, Abcam, Cambridge, England) at $4^{\circ} \mathrm{C}$ overnight. After being washed with PBS three times, the blastocysts or cells were incubated with the fluorescent secondary antibody (1:2000 diluted, Beijing Zhongshan Jinqiao Biotechnology Co., LTD, Beijing, China) in the dark at $37^{\circ} \mathrm{C}$ for $1 \mathrm{~h}$, and then with PI or DAPI at room temperature for $5 \mathrm{~min}$. Finally, they were examined under a laser scanning confocal microscope (TCS SP8 STED; Wetzlar, Hessen, GER).

\section{Western blot}

The proteins were extracted from the frozen blastocysts and EECs with lysis buffer (Nanjing KeyGen Biotech, Nanjing, China). Protein was quantified with the bicinchoninic acid (BCA) protein assay kit (Nanjing KeyGen Biotech). Equal amounts of protein $(20 \mu \mathrm{g})$ were separated via the $10 \%$ sodium dodecyl sulfate polyacrylamide gel electrophoresis and electro-transferred to $0.22 \mu \mathrm{m}$ polyvinylidene difluoride membranes (Millipore, Bedford, MA). After the transfer above, the membranes were blocked in the TBST supplemented with $10 \%$ skim milk at room temperature for $1 \mathrm{~h}$, and incubated with the relevant primary 
antibodies at $4^{\circ} \mathrm{C}$ overnight (Table S6). The next day, the membranes were incubated with the HRPlabeled secondary antibodies (1:5000 dilutions) at room temperature for $1 \mathrm{~h}$. The immunoreactive bands were visualized with ECL reagent under the Gel Imaging System (Tannon Science \& Technology, Shanghai, China) and protein levels digitized with the Quantity One software (Bio-Rad).

\section{Statistical analysis}

The data from the study were presented as the mean \pm standard error of mean from at least triplicate independent experiments. They were analyzed with one-way analysis of variance, followed by Fisher's least significant different test (Fisher LSD) and also the independent samples t-test with the Statistical Package for the Social Sciences (SPSS) software (Version 18.0; SPSS, Chicago, IL). Differences were regarded as being significant at $P<0.05$.

\section{Results}

\section{Expression of IFN $\tau$ mRNA in dairy cow blastocyst}

In this study, the in vivo blastocysts used in the experiments were purchased directly from Beijing Dairy Cow Center. The early blastocysts and expanded ones required for the experiment were continuously obtained with the PA, IVF and SCNT methods (Fig. 1A, B and D), and the blastocyst rates were $32.50 \pm 2.00,31.67 \pm 2.13$ and $6.71 \pm 1.90$, respectively (Table S7). The RT-PCR results show that IFNt mRNA can be expressed in the blastocysts derived from in vivo, PA, IVF and SCNT sources and, with the development and incubation of the embryos, the expression of IFN $\tau$ mRNA was significantly higher in PA, IVF and SCNT expanded blastocysts than in the early blastocysts $(P<0.5)$ (Fig. 1D).

\section{Expression and location of IFNT, IFNAR1 and IFNAR2 in embryos after the co-culture of blastocysts and endometrial epithelial cells}

We used the immunofluorescence method to detect the localization of IFNT protein in each group of blastocysts after the co-culture of blastocysts and EECs. As shown in Fig. 2A, IFNt protein is mainly expressed in the trophoblast cells derived from the in vivo, PA, IVF, and SCNT blastocysts, but not localized or expressed in the inner cell mass. The fluorescence intensity of IFN $\tau$ protein was significantly higher in the SCNT and EECs co-culture group than in the other three groups, and the IFNT protein fluorescence intensity was significantly higher in the in vivo co-culture group with EECs than in the other two groups $(P<0.05)$ (Fig. 2B). Whereafter, we further explored the effect of IFNt secreted by blastocyst trophoblast cells on the expression and localization of its own IFNAR1 and IFNAR2 proteins through autocrine action. In this study, IFNAR1 and IFNAR2 proteins were mainly localized to the trophoblast cells of the embryo (Fig. 2C). The fluorescence intensity of IFNAR1 protein was significantly higher in the SCNT 
and EECs co-culture group than in the other three groups, and that was significantly higher in the in vivo co-culture group with EECs than in the other two groups $(P<0.05)$ (Fig. 2D). However, the fluorescence intensity of the IFNAR2 protein was significantly higher in the in vivo PA and SCNT co-culture group than in the IVF group $(P<0.05)$ (Fig. 2D), but there was no significant difference between the three groups $(P>0.05)$ (Fig. 2D). The localized expression pattern of IFNAR1 protein is consistent with that of IFNT in blastocysts from different sources.

\section{Expression of endometrial receptivity related factors in co- culture of blastocysts and endometrial epithelial cells}

The IFNT secreted by the blastocyst trophoblast cells mainly interacts with the adjacent endometrial epithelial cells (EECs) through paracrine action, so we isolated and identified the bovine EECs and found that both IFNAR1 and IFNAR2 can be expressed in EECs (Fig. S1). After that, the blastocysts of the four groups (Day 7) were co-cultured with EECs for $24 \mathrm{~h}$, and the early blastocysts could develop into expanded or hatched blastocysts (Fig. 3A). Co-cultivation of EECs and blastocysts caused a significant upregulation of the expressions of IFNAR1 and IFNAR2 protein in the EECs $(P<0.05)$ (Fig. 3B, C). At the same time, the co-culture of EECs and blastocysts triggered a significant upregulation of the expressions of Integrin av and Integrin $\beta 3$ protein in the EECs $(P<0.05)$ (Fig. 3B, C). In addition, the RT-PCR results showed that the significant up-regulation of WNT7A mRNA target appeared in the EECs after the coculture of in vivo IVF, PA, SCNT blastocysts and EECs $(P<0.05)$ (Fig. 3D, E). The significant up-regulation of ISG15 mRNA appeared in the EECs after the co-cultivation of IVF, in vivo, SCNT blastocysts and EECs, and the expression of ISG15 mRNA in the EECs after induction by in vivo and SCNT co-cultivation was significantly higher than that induced by IVF co-cultivation in vivo $(P<0.05)$ (Fig. 3D, E). There was no significant expression of ISG15 mRNA in the EECs after the co-cultivation of PA blastocysts and EECs $(P>0.05)$ (Fig. 3D, E,).

\section{Antagonistic blastocyst interferon receptor inhibits the expression of embryonic implantation factors in EECs co- cultured with blastocysts}

IFNAR-IN-1 hydrochloride (IN-1) is an antagonist of IFNAR function. Our results show that $0.01 \mathrm{ng} / \mathrm{mL}$ IFNT stimulated the significant expression of IFNAR downstream factors ISG15 and IRF9 in EECs, which were inhibited by $5 \mu \mathrm{M} \mathrm{IN}-1(P<0.05)$ (Fig. S2A, B). Bovine embryo IFNAR1 mRNA and protein are expressed from the MIl stage to the blastocyst stage, while IFNAR2 mRNA and protein are not expressed from the MII stage to the 4-cell stage, and they are expressed from the 8-cell stage (Fig. 4A, B). Therefore, the antagonist IN-1 was chosen to treat the embryo from the 8-cell stage of the embryo. The expressions of ISG15 and IRF9 were significantly inhibited by both $10 \mu \mathrm{M}$ and $15 \mu \mathrm{M}$ IN-1 treatment $(P<0.01)$ (Fig. S2C, D). In addition, blastocyst rates were significantly reduced by $15 \mu \mathrm{M} I \mathrm{~N}-1$ treatment (Table 4). Co- 
incubation of developmental blastocysts and EECs treated with $10 \mu \mathrm{M} \mathrm{IN-1} \mathrm{significantly} \mathrm{inhibited} \mathrm{the}$ expression of Integrin av, Integrin $\beta 3$ and WNT7A caused by the co-culture of normal blastocysts $(P<0.05)$ (Fig. 4C, D)

\section{Knockout of embryonic INFAR1 and IFNAR2 respectively affects the expression of embryonic implantation factors in co-cultured EECs}

In order to further explore the role of embryonic INFAR1 and IFNAR2 in affecting the expression of cocultured EECs, CRISPR/Cas9 was used to knock out embryonic IFNAR1 and IFNAR2 and co-cultured with EECs to explore the differential expression of Integrin av $\beta 3$ and WNT7A. As shown in Fig. 5A, compared with Standard $1(20 \%)$ and Standard $2(70 \%)$, the viability rates of sgRNA1, sgRNA2, sgRNA3, and sgRNA4 are $50 \%, 25 \%, 30 \%$, and $65 \%$, respectively. Therefore, sgRNA1 and sgRNA4 were selected for subsequent microinjection. The effective sgRNA1 and sgRNA4 were microinjected into the embryos $10 \mathrm{~h}$ after the sperm-egg combination, and then continued to develop to the blastocysts and be incubated with EEC for $48 \mathrm{~h}$. The blastocysts were collected for T7E1 digestion reaction. The RT-PCR results showed that the edited blastocysts with INFAR1 and IFNAR2 genes were successfully obtained. (Fig. 5B). Co-cultured EECs with IFNAR1 and IFNAR2 cleavage were collected in the blastocyst genome and qRT-PCR detection results showed that the expressions of Integrin av and WNT7A mRNA are significantly lower in the cocultured group of IFNAR1 and IFNAR2 gene-edited blastocysts and EEC than in normal blastocysts and EECs cocultivation. The expression of Integrin av mRNA was significantly lower in the IFNAR2 gene-edited blastocyst co-culture group with EEC than in the IFNAR1 co-culture group with EEC. The expression of Integrin $\beta 3$ mRNA was significantly lower in IFNAR2 gene-edited blastocysts and EEC co-culture group than in the normal blastocysts and IFNAR1 gene-edited blastocysts and EEC co-culture group, while the Integrin $\beta$ mRNA expression was not significantly different in IFNAR1 gene-edited blastocysts and EEC coculture group from that in the normal blastocyst and EEC co-culture group $(P<0.05)$ (Fig. 5C).

\section{Knockout of IFNAR in EECs affects the expression of implantation factors in co-cultured EECs}

EECs are the main target cells for IFN $\tau$ secreted by blastocyst trophoblast cells and express both IFNAR1 and IFNAR2. Therefore, CRISPR/Cas9 was used to knock out IFNAR in the EECs to obtain IFNAR1/IFNAR2 double knockout cell lines in this study (Fig. S3A-D). After that, the expressions of Integrin av $\beta 3$ and WNT7A protein were significantly lower in the IFNAR1'/IFNAR2 ${ }^{-}$and IVF co-culture group than in the normal blastocyst and EEC co-culture group. The expression of WNT7A protein was significantly higher in the IFNAR1\%/IFNAR2 ${ }^{-}$and IVF co-culture group than in IFNAR1'/IFNAR2 cells, while the expressions of Integrin av and Integrin $\beta 3$ protein did not increase significantly $(P<0.05)$ (Fig. 6A, B). Meanwhile, the expressions of Integrin av $\beta 3$ and WNT7A protein were significantly lower in the 
IFNAR1 ${ }^{-} /$IFNAR2 ${ }^{-}$and PA co-culture group than in the normal blastocyst and EEC co-culture group. Compared with IFNAR1\%/IFNAR2 cells, the expression of Integrin av, Integrin $\beta 3$ and WNT7A protein did not increase significantly in the IFNAR1 ${ }^{\circ} /$ IFNAR2 ${ }^{-}$and PA co-culture group $(P<0.05)$ (Fig. 6C, D).

\section{Knockout of embryonic INFAR1 and IFNAR2 affects the expression of implantation factors in co-cultured EECs}

In order to further explore the role of INFAR1 and IFNAR2 in EECs in affecting the expression of implantation factors in co-cultured EECs. CRISPR/Cas9 was used to obtain IFNAR1 and IFNAR2 knockout cell lines (Fig. S4A, B), and then co-cultured with IVF and PA blastocysts to explore the differential expression of Integrin av $\beta 3$ and WNT7A (Fig. 7A, B). Our results found that the expressions of Integrin av $\beta 3$ mRNA were significantly lower in the co-culture group of EEC (C8, IFNAR1 $\%$ IFNAR2 ${ }^{+}$) and EEC (D5, IFNAR $1^{+} /$IFNAR2 $)$and IVF than in the co-culture group of EEC (IFNAR $1^{+} /$IFNAR2 $\left.{ }^{+}\right)$and IVF $(P<0.05)$ (Fig. 7C), whereas the expression of WNT7A did not differ significantly in each group ( $P>0.05$ ) (Fig. 7C). PA cocultured with EEC (IFNAR1 ${ }^{+} /$IFNAR2 $\left.{ }^{+}\right)$, EEC (C8, IFNAR1 $\%$ IFNAR2 ${ }^{+}$) and EEC (D5, IFNAR1 ${ }^{+} /$IFNAR2 $)$did not show significant differences from embryo implantation factors ( $P>0.05)$ (Fig. 7D).

\section{Discussion}

Embryo transfer technology has been successfully applied to livestock for more than 40 years, but its pregnancy rate has been basically stable, but between 50 and $60 \%$. People have done a lot of research to improve the transplantation technology, but it is still difficult to achieve a major breakthrough and embryos prepared in vitro are different in quality from those produced by natural mating of the mother under normal conditions [18]. In addition, the embryo transfer rate of somatic cell cloning in vitro is also at a lower level [19]. Adopted in this study, the in vitro bovine embryo production system has been used by the research team for many years, and can guarantee the stable production of in vitro blastocysts that meet the specific experimental requirements, but has not solved the problem of a lower in vitro blastocyst rate, which may also be the internal reason for final reduced pregnancy rates. Many studies have found that IFN- $\tau$ secreted from the conceptus by increasing the expression of ISG in the cervix and vagina can safeguard the development of blastocyst in the uterus [20]. Our research found the expression and location of IFNT in blastocysts from different sources and that with the development and incubation of the embryos, the expression of IFNT mRNA was significantly higher in PA, IVF and SCNT expanded blastocysts than in the early blastocysts. In addition, IFNT, IFNAR1 and IFNAR2 are all located in the trophoblast cells of the blastocyst. Furthermore, the fluorescence intensity of IFNAR1 is consistent with IFNT, suggesting that IFNAR1 mainly plays a major role in the IFNT autocrine regulation of blastocyst development. Also other factors affect the bovine embryo development in vitro, such as insulin-like growth factor-1 (IGF-1), colony stimulating factor-2 (CSF-2) and hyaluronan [21]. Related research also confirmed that IFNAR1 is expressed in the trophectoderm of ovine conceptuses on the 15th and 20th days [22]. IFNAR1 mainly distributes in chorioallantoic membrane, endometrial epithelium, cotyledon and 
caruncle during the bovine early pregnancy and changes depending on time and position. The IFNAR1 and IFNAR2 expressions were mainly detected in chorioallantoic membrane and cotyledon, and markedly increased along with the pregnant process. Moreover, the expression level of IFNAR1 was higher than that of IFNAR2 in chorioallantoic membrane and cotyledon [13]. In this study, it was also found that IFNAR1 was more involved in the development of the embryo. By contrast, IFNAR2 has been shown to play an important role in HIV invasion and immune regulation $[14,15]$. As a foreign body relative to the mother, the embryo also triggers immune tolerance in the process of establishing contact with the endometrium. Some studies confirmed that gestational status does not affect IFNAR1 or IFNAR2 expression, but IFNAR2 showed a distinct response between PMNs and PBMCs of pregnant heifers [14]. In this study, it was found that IFNAR2 was more involved in directing normal uterine preparation for bovine embryo implantation.

It is the prerequisite for the establishment of a precise dialogue between embryo and uterus to establish a connection between embryonic trophoblast cells and EEC tissue structure. Many studies have reported that the co-culture of embryonic trophoblast cells or blastocysts with endometrial epithelial monolayer cells in vitro can be used to explore the interactions between blastocysts and uterus in vivo, including blastocyst adhesion, blastocyst development, and endometrial epithelial cell migration, and related factors' expression [23-25]. Therefore, we successfully constructed a co-culture model of blastocystEECs, with which, the co-culture of bovine blastocysts and their EECs from different sources stimulated the expressions of IFNAR1, IFNAR2 and its downstream Integrin av $\beta 3$, WNT7A, and ISG15 genes. Our results are consistent with existing reports that endometrial cells are largely in response to IFN $\tau$ actions and possibly associated with the crucial biological processes during the peri-implantation period of pregnancy [26].

In order to further explore the roles of IFNAR1 and IFNAR2 in regulating the dialogue between blastocysts and uterus, we firstly found that the expression of IFNAR1 runs through the development process from oocytes to the entire blastocyst, while that of IFNAR2 only appeared when it developed to 8-cell embryo, indicating that IFNAR2 plays a major role in establishing the dialogue between the late blastocyst and the uterus. Antagonizing the expression of IFNAR1 and IFNAR2 in embryos and co-culture with EECs also reduced the expression of Integrin av $\beta 3$, WNT7A, ISG15 genes in EECs. After knocking out IFNAR1 and IFNAR2 with CRISPER-cas9, it was found from the co-cultivation with EECs that although both can reduce the expression of Integrin av $\beta 3$ and WNT7A in EECs, deletion of the IFNAR2 gene has a greater impact than that of the IFNAR1 gene.

Next, the CRISPER-cas9 method was used to prepare IFNAR1 and IFNAR2 double knockout cell lines and single knockout cell lines in this study. According to our results, the expression of Integrin av $\beta 3$ was inhibited when the IFNAR1 and IFNAR2 double knockout cell lines were co-cultured with IVF and PA embryos. However, the expression of WNT7A was not inhibited in IVF co-culture. The expression of Integrin av $\beta 3$ was inhibited, the inhibition of IFNAR1 ${ }^{+} /$IFNAR2 $^{-}$was stronger, and the expression of WNT7A was not inhibited when IFNAR1-/IFNAR2 ${ }^{+}$and IFNAR1 ${ }^{+} /$IFNAR2 ${ }^{-}$were co-cultured with IVF. The expression of Integrin av $\beta 3$ and WNT7A did not change significantly when IFNAR1 ${ }^{-} /$IFNAR2 ${ }^{+}$and 
IFNAR $1^{+} /$IFNAR2- were co-cultured with PA. Existing studies have found that WNT7A presented with a significant decrease between embryo transfer and pregnancy testing in case of ongoing pregnancy, women with implantation failure showed unaltered concentrations [27]. Therefore, WNT7A might be a key factor leading to a lower cleavage rate and a lower pregnancy rate of blastocysts obtained in vitro. Studies have reported that compared with embryos in vivo, parthenogenetic embryos have a higher rate of chromosomal ploidy abnormalities, and there are differences in the expression of protein factors [28, 29]. The lack of paternal genes in parthenogenetically activated embryos may also result in their inability. Related studies have already reported sexual dimorphism in IGF1 gene expression, embryonic functional regulation and developmental potential [30].

\section{Conclusions}

In summary, IFNT secreted from bovine blastocysts regulates its own development through autocrine action in combination with IFNAR1 and the establishment of endometrial receptivity through paracrine action in combination with IFNAR2, and activates the expression of the Integrin av, Integrin $\beta 3$, WNT7A and ISG15 cascade reaction. As a result, the bovine embryo-uterine dialogue is regulated precisely (Fig. 8). In addition, WNT7A might be a key factor leading to a lower cleavage rate and a lower pregnancy rate of the blastocysts obtained in vitro relative to in vivo.

\section{Abbreviations}

IFNT: Interferon-tau; IFNAR: IFNT receptor; SCNT: Somatic cell nuclear transfer; IVF: In vitro fertilization; PA: Parthenogenetic activation; EEC: endometrial epithelial cell; WNT7A: WNT family subtype glycoprotein 7A; ISG15: interferon stimulated gene 15; E2: Estrogen; P4: progesterone; MUC-1: Mucin-1; IGF-1: insulin-like growth factor-1; CSF-2: colony stimulating factor-2; IN-1: IFNAR-IN-1 hydrochloride.

\section{Declarations}

\section{Acknowledgements}

We would like to express our great appreciation to Sarah Williams, PhD, from Liwen Bianji, Edanz Group China (www.liwenbianji.cn), for editing the English draft of this manuscript.

\section{Authors' contributions}

Xiangguo Wang: Validation, Data Curation, Writing - Original Draft, Visualization, Chaolei Chen: Investigation, Resources, Lijuan Wang: Investigation, Resources, Yunze Su: Investigation, Resources, Boyu Li: Investigation, Resources, Longfei Xiao: Writing - Review \& Editing, Supervision, Zili Lin: Writing - Review \& Editing, Xihui Sheng: Writing - Review \& Editing, Xiaolong Qi: Formal analysis, 
Hemin Ni: Supervision, Yong Guo: Conceptualization, Methodology, Writing - Review \& Editing, Funding acquisition.

\section{Funding}

This study was supported by the National Fund for Natural Sciences of China (Grant no. 31772697 , 31802263) and 2016 Beijing Municipal Science and Technology Innovation Service Capacity Building Project (Grant no. KZ201610020018).

\section{Availability of data and materials}

The datasets used and analysed during the current study are available from the corresponding author on reasonable request

\section{Ethics approval and consent to participate}

This study was performed in accordance with the Guidelines of the Animal Ethic Committee of Beijing University of Agriculture under the permit No. SYXK (JING) 2015-0004.

\section{Consent for publication}

Not applicable

\section{Competing interests}

The authors declare that they have no competing interests.

\section{Author details}

${ }^{1}$ Animal Science and Technology College, Beijing University of Agriculture, Beijing 102206, China

\section{References}

1. Paria BC, Reese J, Das SK, Dey SK: Deciphering the cross-talk of implantation: advances and challenges. Science2002, 296:2185-2188.

2. Dey SK, Lim H, Das SK, Reese J, Paria BC, Daikoku T, Wang H: Molecular cues to implantation. Endocr Rev2004, 25:341-373. 
3. Wang H, Dey SK: Roadmap to embryo implantation: clues from mouse models. Nat Rev Genet2006, 7:185-199.

4. Spencer TE, Sandra O, Wolf E: Genes involved in conceptus-endometrial interactions in ruminants: insights from reductionism and thoughts on holistic approaches. Reproduction2008, 135:165-179.

5. Spencer TE, Forde N, Lonergan P: The role of progesterone and conceptus-derived factors in uterine biology during early pregnancy in ruminants. J Dairy Sci2016, 99:5941-5950.

6. Hantak AM, Bagchi IC, Bagchi MK: Role of uterine stromal-epithelial crosstalk in embryo implantation. Int J Dev Bio/2014, 58:139-146.

7. Bazer FW, Burghardt RC, Johnson GA, Spencer TE, Wu G: Interferons and progesterone for establishment and maintenance of pregnancy: interactions among novel cell signaling pathways. Reprod Bio/2008, 8:179-211.

8. Roberts RM, Chen Y, Ezashi T, Walker AM: Interferons and the maternal-conceptus dialog in mammals. Semin Cell Dev Biol2008, 19:170-177.

9. Bazer FW, Spencer TE, Johnson GA, Burghardt RC, Wu G: Comparative aspects of implantation. Reproduction2009, 138:195-209.

10. Ealy $A D$, Yang $Q E$ : Control of interferon-tau expression during early pregnancy in ruminants. $A m J$ Reprod Immuno/2009, 61:95-106.

11. Rosenfeld CS, Han CS, Alexenko AP, Spencer TE, Roberts RM: Expression of interferon receptor subunits, IFNAR1 and IFNAR2, in the ovine uterus. Biol Reprod2002, 67:847-853.

12. Lonergan $P$, Forde $N$ : Maternal-embryo interaction leading up to the initiation of implantation of pregnancy in cattle. Anima/2014, 8 Suppl 1:64-69.

13. Wang W, Liu R, Liang X, Zhao Q, Qu P, Yao K, Jiang M, Luo Y, Zhang W, Qing S: Expression of IFNAR1 and IFNAR2 in cattle placenta during early pregnancy. Reprod Domest Anim2018, 53:385-392.

14. Melo GD, Pinto LMF, Rocha CC, Motta IG, Silva LA, da Silveira JC, Gonella-Diaza AM, Binelli M, Pugliesi G: Type I interferon receptors and interferon-tau-stimulated genes in peripheral blood mononuclear cells and polymorphonuclear leucocytes during early pregnancy in beef heifers. Reprod Fertil Dev2020, 32:953-966.

15. Qing J, Wu M, Luo R, Chen J, Cao L, Zeng D, Shang L, Nong J, Wu Q, Ding BS, et al: Identification of Interferon Receptor IFNAR2 As a Novel HCV Entry Factor by Using Chemical Probes. ACS Chem Bio/2020, 15:1232-1241.

16. Hernandez N, Bucciol G, Moens L, Le Pen J, Shahrooei M, Goudouris E, Shirkani A, Changi-Ashtiani M, Rokni-Zadeh H, Sayar EH, et al: Inherited IFNAR1 deficiency in otherwise healthy patients with adverse reaction to measles and yellow fever live vaccines. J Exp Med2019, 216:2057-2070.

17. Bao ZJ, Zhao S, Haq IU, Zeng SM: Recombinant bovine interferon-tau enhances in vitro development of bovine embryos by upregulating expression of connexin 43 and E-cadherin. J Dairy Sci2014, 97:6917-6925. 
18. Ferre LB, Kjelland ME, Taiyeb AM, Campos-Chillon F, Ross PJ: Recent progress in bovine in vitroderived embryo cryotolerance: Impact of in vitro culture systems, advances in cryopreservation and future considerations. Reprod Domest Anim2020, 55:659-676.

19. Czernik M, Anzalone DA, Palazzese L, Oikawa M, Loi P: Somatic cell nuclear transfer: failures, successes and the challenges ahead. Int J Dev Bio/2019, 63:123-130.

20. Kunii H, Koyama K, Ito T, Suzuki T, Balboula AZ, Shirozu T, Bai H, Nagano M, Kawahara M, Takahashi M: Hot topic: Pregnancy-induced expression of interferon-stimulated genes in the cervical and vaginal mucosal membranes. J Dairy Sci2018, 101:8396-8400.

21. Block J, Hansen PJ, Loureiro B, Bonilla L: Improving post-transfer survival of bovine embryos produced in vitro: actions of insulin-like growth factor-1, colony stimulating factor-2 and hyaluronan. Theriogenology2011, 76:1602-1609.

22. Wang $\mathrm{XL}$, Wang $\mathrm{K}$, Han GC, Zeng SM: A potential autocrine role for interferon tau in ovine trophectoderm. Reprod Domest Anim2013, 48:819-825.

23. Li HY, Shen JT, Chang SP, Hsu WL, Sung YJ: Calcitonin promotes outgrowth of trophoblast cells on endometrial epithelial cells: involvement of calcium mobilization and protein kinase $\mathrm{C}$ activation. Placenta2008, 29:20-29.

24. Gomez E, Carrocera S, Martin D, Sanchez-Calabuig MJ, Gutierrez-Adan A, Murillo A, Munoz M: Hepatoma-derived growth factor: Protein quantification in uterine fluid, gene expression in endometrial-cell culture and effects on in vitro embryo development, pregnancy and birth. Theriogenology2017, 96:118-125.

25. Wang X, Zhu B, Xiong S, Sheng X, Qi X, Huang Q, Chen C, Guo Y, Ni H: Expression and function of MUC1 in uterine tissues during early pregnancy in sheep after natural oestrous or artificially-induced oestrous. Theriogenology2018, 108:339-347.

26. Boruszewska D, Kowalczyk-Zieba I, Sinderewicz E, Grycmacher K, Staszkiewicz J, Woclawek-Potocka I: The effect of lysophosphatidic acid together with interferon tau on the global transcriptomic profile in bovine endometrial cells. Theriogenology2017, 92:111-120.

27. Freis A, Keller A, Ludwig N, Meese E, Jauckus J, Rehnitz J, Capp E, Strowitzki T, Germeyer A: Altered miRNA-profile dependent on ART outcome in early pregnancy targets Wnt-pathway. Reproduction2017, 154:799-805.

28. Hyttel P, Viuff D, Laurincik J, Schmidt M, Thomsen PD, Avery B, Callesen H, Rath D, Niemann H, Rosenkranz C, et al: Risks of in-vitro production of cattle and swine embryos: aberrations in chromosome numbers, ribosomal RNA gene activation and perinatal physiology. Hum Reprod2000, 15 Suppl 5:87-97.

29. Niemann H, Wrenzycki C: Alterations of expression of developmentally important genes in preimplantation bovine embryos by in vitro culture conditions: implications for subsequent development. Theriogenology2000, 53:21-34.

30. Tribulo P, Jumatayeva G, Lehloenya K, Moss JI, Negron-Perez VM, Hansen PJ: Effects of sex on response of the bovine preimplantation embryo to insulin-like growth factor 1 , activin A, and WNT7A. 


\section{Figures}

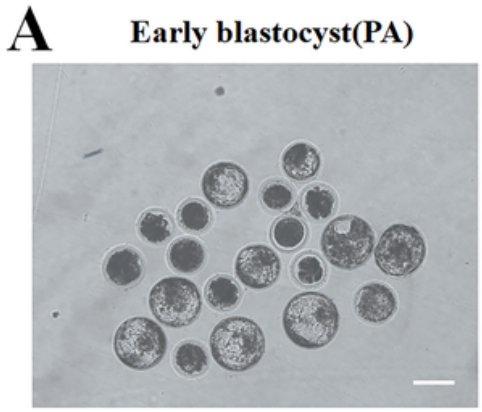

B

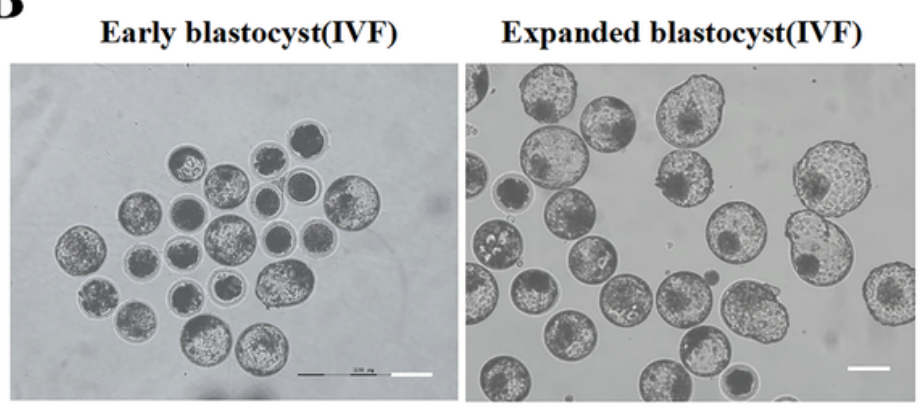

Expanded blastocyst(PA)

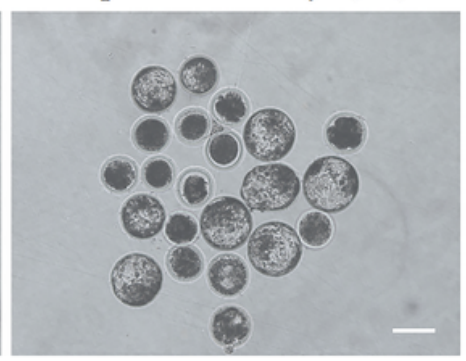

D IFN- $\tau^{-1}$

$\beta$-actin
Expanded blastocyst(SCNT)
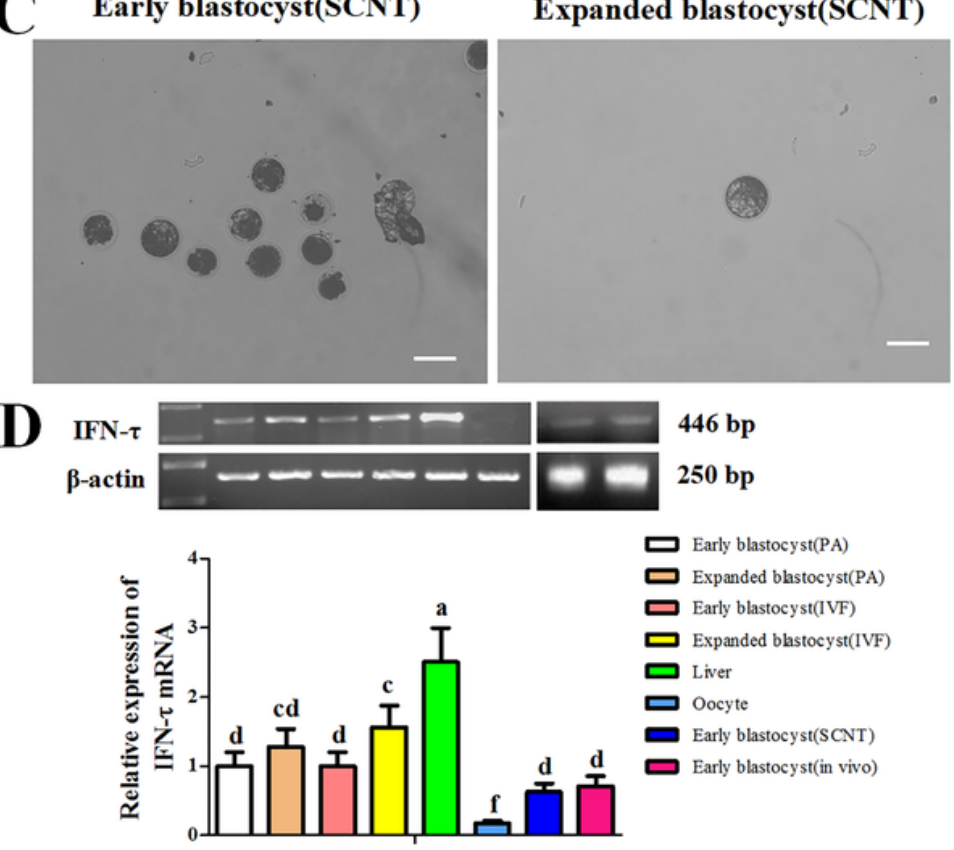

\section{Figure 1}

Expression of IFNT mRNA in the blastocyst of the dairy cows. (A) The oocytes expelled from the first polar body were moved into the ION droplets to avoid light activation and cultured to obtain PA early blastocysts and expanded blastocysts. Representative images of five independent experiments are shown (Scale bar: $100 \mu \mathrm{m}$ ). (B) In vitro mature oocytes and Limousin bovine semen (No. 11108016) were fertilized in vitro to obtain IVF early blastocysts and expanded blastocysts. Representative images of five independent experiments are shown (Scale bar: $100 \mu \mathrm{m}$ ). (C) Bovine ear fibroblasts were injected into the enucleated oocytes and cultured to obtain SCNT early blastocysts and expanded blastocysts.

Representative images of five independent experiments are shown (Scale bar: $100 \mu \mathrm{m}$ ). (D) RT-PCR was used to detect the expression of IFNt in the blastocyst derived from PA, IVF, SCNT, and in vivo, with liver tissue as a positive control and oocytes as a negative control. Data are presented as the mean \pm standard error of mean. Values with different superscripts are significantly different $(P<0.05)$. The experiments were replicated three times. PA: parthenogenetic activation; SCNT: somatic cell nuclear transfer; IVF: in vitro fertilization.

\section{Figure 2}


Expression and location of IFNT, IFNAR1 and IFNAR2 in the embryos after the co-culture of blastocysts and endometrial epithelial cells (EECs). (A\&C) Blastocysts in the four groups (Day \#7) were co-cultured with EECs for $24 \mathrm{~h}$. After that, PI, IFNT, IFNAR1 and IFNAR2 staining were performed on the blastocysts in the four groups. Each sample was counterstained with PI to visualize DNA (red). Representative images of five independent experiments are shown $(n=5)($ Scale bar: $50 \mu \mathrm{m})$. (B\&D) Quantification of IFNt/DNA, IFNAR1/DNA and IFNAR2/DNA signal intensities in the blastocysts in the four groups. Labeling intensity was expressed relative to that of the in vivo group (set as 1.0). Values with different superscripts differ significantly $(a, b, \& c, P<0.05)$. The experiments were replicated three times. In each of the replications, $n$ = 8-10 per group. PI: Propidium lodide; PA: parthenogenetic activation; SCNT: somatic cell nuclear transfer; IVF: in vitro fertilization. 


\section{A}

\section{Figure 3}

Expression of endometrial receptivity-related factors in the co-culture of blastocysts and endometrial epithelial cells (EECs). (A) Blastocysts in the four groups (Day \#7) were co-cultured with EECs for $24 \mathrm{~h}$ (Scale bar: $500 \mu \mathrm{m}$ ). (B) Co-cultured EECs were collected, and total protein from EECs was subjected to the detection of the expressions of Integrin av, Integrin $\beta 3$, IFNAR1 and IFNAR2 with the western blot method. $\beta$-actin served as the control gene in the all samples. Representative images of three 
independent experiments are shown. (C) Relative expression of each protein was performed by grayscale analysis. The data are presented as mean \pm S.D. of three independent experiments. Bars with different letters are significantly different $(a, \& b, P<0.05)$. (D) Total RNA from EECs was subjected to the detection of the expression of WNT7A and ISG15 with the RT-PCR method. $\beta$-actin served as the control gene in the all samples. Representative images of three independent experiments are shown. (E) Relative expression of each mRNA was performed by grayscale analysis. The data are presented as the mean \pm S.D. of three independent experiments. Bars with different letters are significantly different $(a, b, \& c, P<0.05)$.
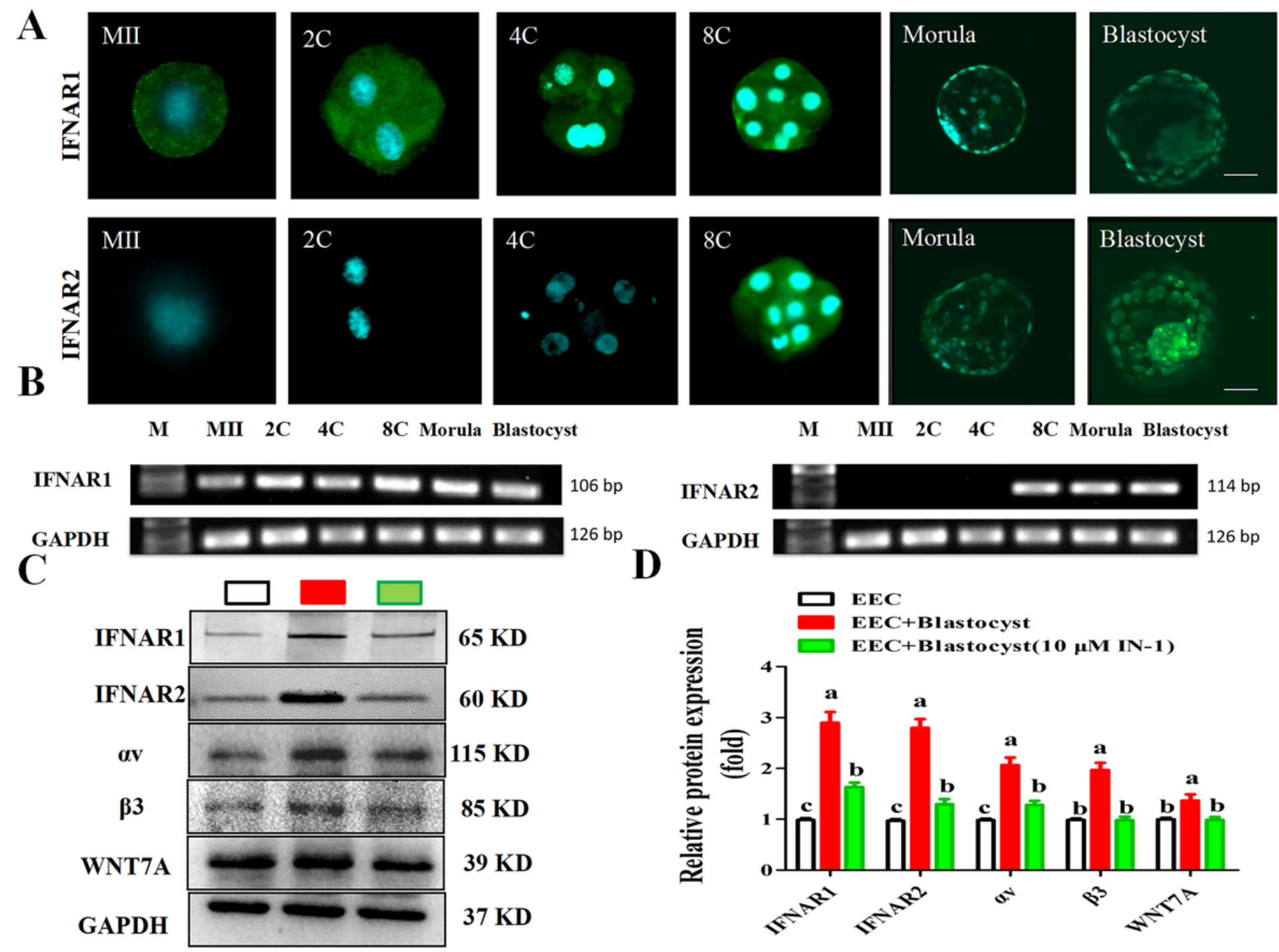

Figure 4

Antagonistic blastocyst interferon receptor inhibits the expression of embryonic implantation factors in EECs co-cultured with blastocysts. (A) In vitro mature cultured oocytes and embryos were collected that developed to the 2-cell, 4-cell, 8-cell, morula and blastocyst stage after artificial fertilization. After that, DAPI, IFNAR1 and IFNAR2 staining was performed on the blastocysts. Each sample was counterstained with DAPI to visualize DNA (blue). Representative images of five independent experiments are shown $(n=5)($ Scale bar: $100 \mu \mathrm{m})$. (B) RT-PCR was used to detect the expressions of IFNAR1 and IFNAR2 in the 
mature oocytes and 2-cell, 4-cell, 8-cell, morula and blastocyst. Representative images of five independent experiments are shown $(n=5)$. (C) 8-cell embryos were incubated with $10 \mu \mathrm{M} \mathrm{IN-1}$ to the blastocyst stage, and then incubated with EECs for $48 \mathrm{~h}$. Co-cultured EECs were collected and total protein from EECs was subjected to the detection of the expressions of IFNAR1, IFNAR2, Integrin av, Integrin $\beta 3$ and WNT7A with the western blot method. $\beta$-actin served as the control gene in the all samples. Representative images of three independent experiments are shown. (D) Relative expression of each protein was performed by grayscale analysis. The data are presented as the mean \pm S.D. of three independent experiments. Bars with different letters are significantly different $(a, \& b, P<0.05)$.

\section{Figure 5}

Knockout of embryonic INFAR1 and IFNAR2 and its effect on the expression of implantation factors in cocultured EECs. (A\&B) The designed sgRNA1-F, sgRNA2-F, sgRNA3-F, sgRNA4-F, and sgRNA-R were biosynthesized, amplified in vitro, purified by phenol/chloroform, and then combined with the whole genome of EECs after the sgRNA1, sgRNA2, sgRNA3, and sgRNA4 targets were amplified for spCas9 activity in vitro biopsy. RT-PCR was used to detect the target cleavage activity. The effective sgRNA1 and sgRNA4 were microinjected into the embryo after $10 \mathrm{~h}$ of sperm-egg combination and continued to develop to the blastocyst. After that, the blastocysts were incubated with EECs for $48 \mathrm{~h}$ and collected for T7E1 digestion reaction. RT-PCR was used to detect the genomic target cleavage activity. (C) Co-cultured EECs with IFNAR1 and IFNAR2 cleavage in the blastocyst genome were collected and qRT-PCR was used to detect the expression of $a v, \beta 3$ and WNT7A mRNA. The data are presented as the mean \pm S.D. of three independent experiments. Bars with different letters are significantly different $(a, \& b, P<0.05)$.

\section{Figure 6}

Knockout of IFNAR in endometrial epithelial cells (EECs) affects the expression of implantation factors in co-cultured EECs. CRISPR/Cas9 was used to knock out IFNAR in EECs to obtain IFNAR1'/IFNAR2- double knockout cell lines, which were co-cultured with IVF $(A)$ and $P A(C)$ blastocysts for $48 \mathrm{~h}$. Co-cultured EECs were collected, and total protein from EECs was subjected to the detection of the expressions of Integrin av, Integrin $\beta 3$ and WNT7A with the western blot method. $\beta$-actin served as the control gene in the all samples. Representative images of three independent experiments are shown. (B\&D) Relative expression of each protein was performed by grayscale analysis. The data are presented as the mean \pm S.D. of three independent experiments. Bars with different letters are significantly different $(a, \& b, P<0.05)$. 


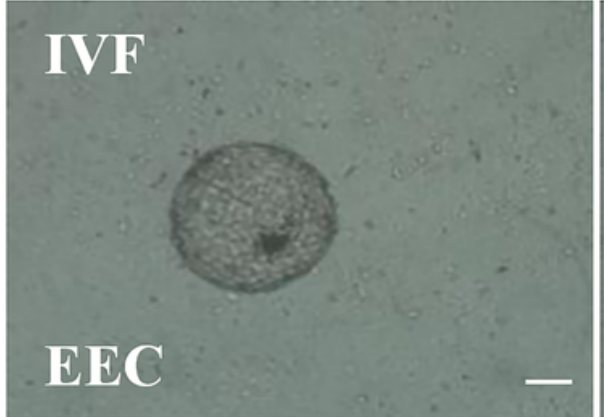

\section{B}
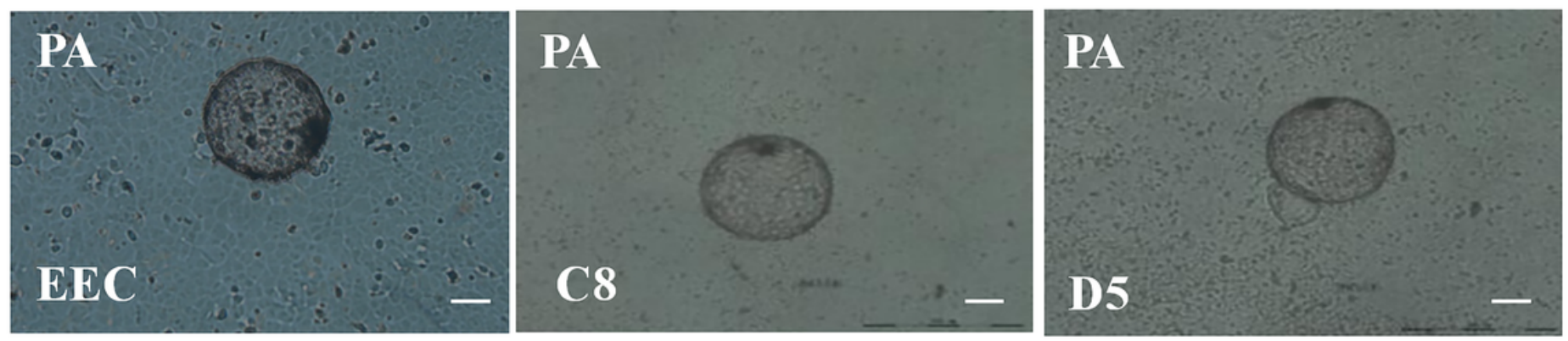

C

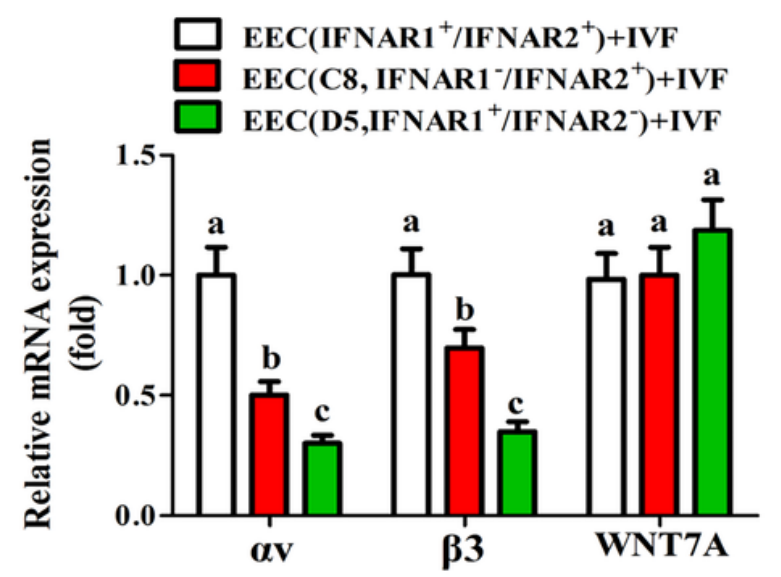

D

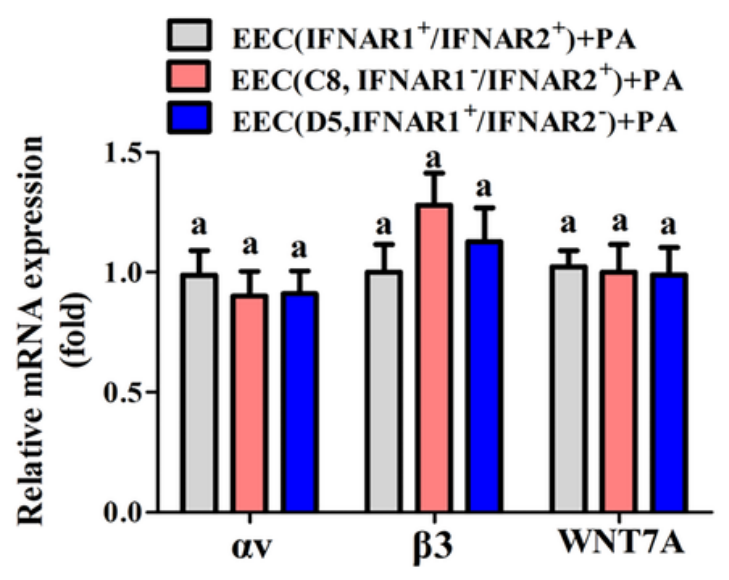

Figure 7

Knockout of INFAR1 and IFNAR2 in endometrial epithelial cells (EECs) and its effect on the expression of implantation factors in EECs and blastocyst co-culture system. (A\&B) IVF and PA were co-cultured with EEC (IFNAR $1^{+} /$IFNAR2 $\left.{ }^{+}\right)$, EEC (C8, IFNAR1\%/IFNAR2 $\left.{ }^{+}\right)$and EEC (D5, IFNAR1 ${ }^{+} /$IFNAR2 $\left.{ }^{-}\right)$separately.

Representative images of five independent experiments are shown $(n=5)($ Scale bar: $100 \mu \mathrm{m})$. (C\&D) After $48 \mathrm{~h}$ of incubation, co-cultured EECs were collected, and total RNA from EECs was subjected to the detection of the expressions of Integrin av, Integrin $\beta 3$ and WNT7A with the qRT-PCR method. $\beta$-actin served as the control gene in all samples. The data are presented as the mean \pm S.D. of three independent experiments. Bars with different letters are significantly different $(a, \& b, P<0.05)$. 


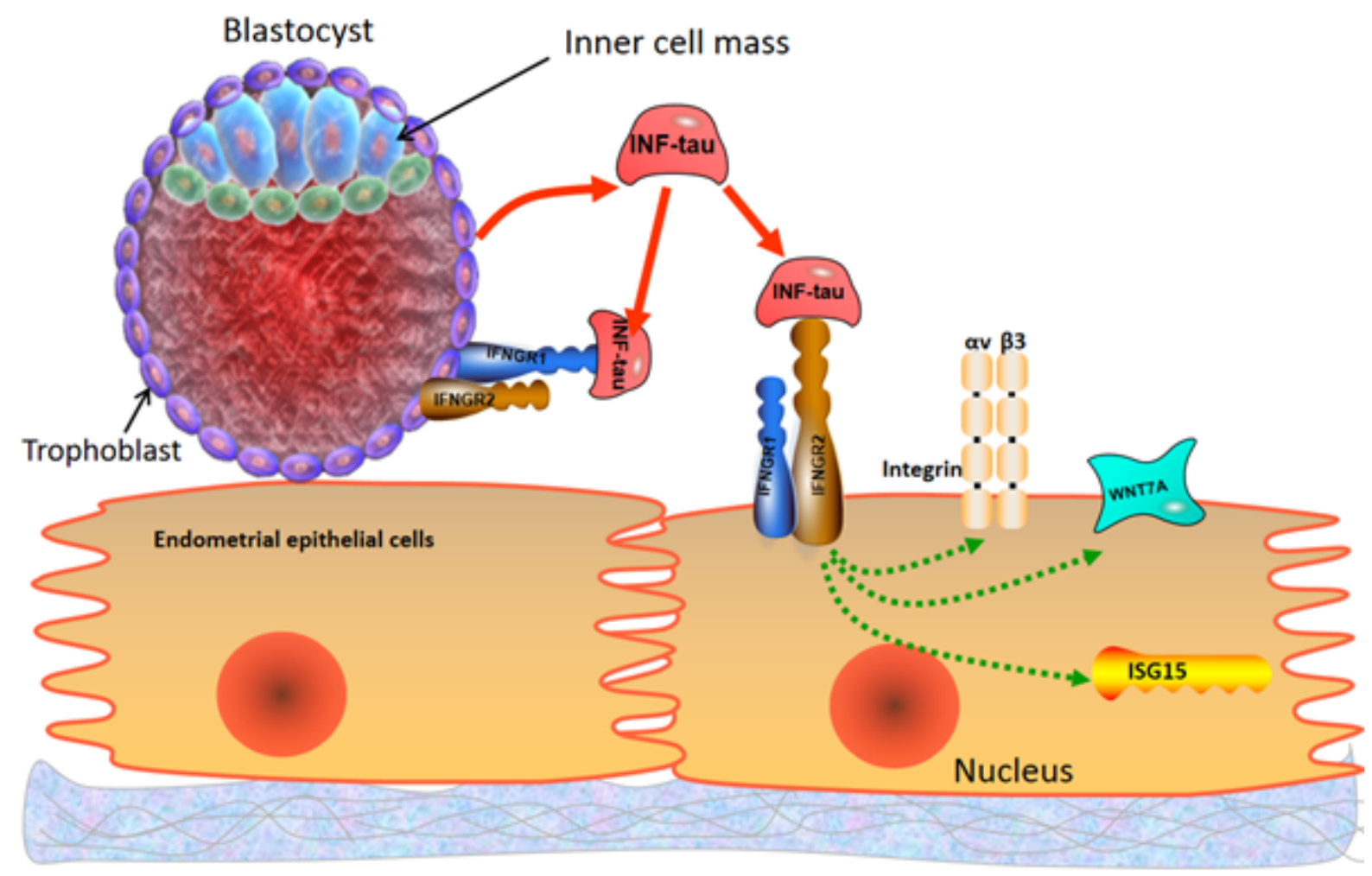

Figure 8

Schematic diagrams show that IFNT secreted by blastocysts regulates its own development through autocrine action in combination with IFNAR1, and the establishment of endometrial receptivity through the paracrine action in combination with IFNAR2, and activates the expressions of the Integrin av, Integrin $\beta 3$, WNT7A and ISG15 cascade reaction. As a reuslt, the embryo-uterine dialogue is regulated precisely.

\section{Supplementary Files}

This is a list of supplementary files associated with this preprint. Click to download.

- supplementarydata.doc 